\title{
AVALIAÇÃO DO EFEITO DO SILICATO DE CÁLCIO E MAGNÉSIO NA PRODUTIVIDADE DO ARROZ (Oryza sativa L.) EM ROTAÇÃO DE CULTURA COM SOJA (Glycine max (L.) Merrill) EM SISTEMA DE PLANTIO DIRETO
}

\author{
Gustavo de Andrade Bezerra'; Neidelane de Alencar Sobrinho'; ${ }^{1}$ Wilson Araújo da \\ Silva $^{2}$; Ivaneide de Oliveira Nascimento ${ }^{2}$; Gisele Barata da Silva ${ }^{3}$; Marta Cristina Corsi \\ de Filippi ${ }^{4}$.

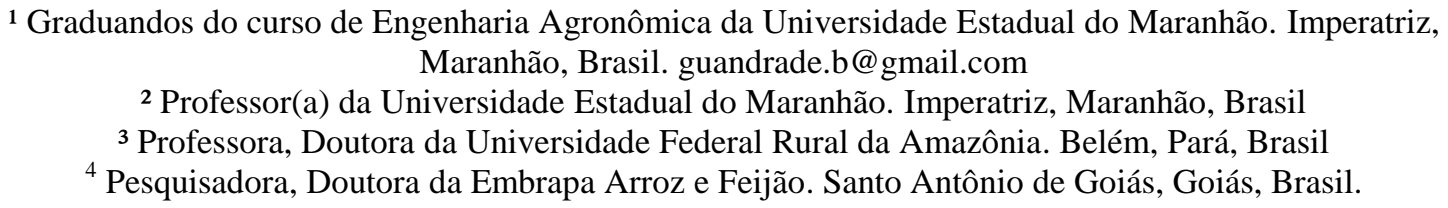

RESUMO: O objetivo foi avaliar o efeito do silicato de cálcio e magnésio na produtividade do arroz em rotação com soja em sistema de plantio direto no estado do Maranhão. $\mathrm{O}$ experimento foi implantado em área do Sindicato Rural de Imperatriz - Parque de exposições. Inicialmente realizou-se coleta e análise de solos para fins de fertilidade. Após os resultados da análise de solo, aplicou-se agrosilício (silicato de cálcio e magnésio) e calcário para correção de acidez do solo. Passado trinta dias, microbiolizou-se 2,6 kg de sementes com rizobactérias promotoras de crescimento (Pseudomonas sp. e Burlkoderia sp.) e procedeu-se o plantio do arroz, em esquema experimental de blocos ao acaso com parcelas subsubdivididas com quatro repetições com cinco tratamentos $\left(0,1,2,4,8\right.$ t.ha ${ }^{-1}$ de silicato de cálcio e magnésio - Agrosilício). Os primeiros resultados foram coletados considerando a emergência e altura de plântulas, seguidas das avaliações de Produtividade, Biomassa e Massa de 100 grãos cheios. Segundo os dados analisados, as dosagens de agrosilício não obtiveram influência sobre o crescimento de plântulas, biomassa, massa de 100 grãos cheios e produtividade do arroz em sistema de plantio direto.

PALAVRAS-CHAVE: adubação, agrosilício, taxa de germinação.

\section{EFFECTS OF CALCIUM AND MAGNESIUM SILICATE IN PRODUCTIVITY OF RICE (Oryza sativa L.) ROTATION OF CULTURE WITH SOYBEAN (Glycine max (L.) Merrill) SYSTEM OF PLANTING IN DIRECT}

\begin{abstract}
The objective was to evaluate the effect of calcium silicate and magnesium yield of rice in rotation with soybeans in no-tillage system in the state of Maranhão. The experiment was established in the area of Rural Union of Imperatriz - Exhibition Park. Initially held collection and analysis for the purpose of soil fertility. After the results of soil analysis, applied agrosilício (silicate of calcium and magnesium) and limestone to correct soil acidity. Last thirty days, microbiolization by $2.6 \mathrm{~kg}$ of seeds with growth promoting rhizobacteria (Pseudomonas sp. Burlkoderia and sp.) and proceeded to planting rice in experimental design of randomized blocks with split plots with four repetitions with five levels $\left(0,1,2,4,8 \mathrm{t} \mathrm{ha}^{-1}\right.$ of calcium silicate and magnesium - Agrosilício). The first results were collected from the emergence and seedling height, followed by reviews of Productivity, Biomass and Mass 100 filled grains. According to
\end{abstract}


the data analyzed, dosages agrosilício not had influence on seedling growth, biomass, 100 filled grains and yield of rice in no-tillage system.

KEY-WORDS: agrosilício, fertilization, germination rate.

\section{INTRODUÇÃO}

O arroz (Oryza sativa L.) é um dos cereais de maior consumo em todo mundo, possui características que lhe proporcionam ser um dos alimentos com melhor balanceamento nutricional, fornecendo $20 \%$ da energia e $15 \%$ da proteína per capita necessária ao homem. Sendo uma cultura extremamente versátil, que se adapta a diferentes condições de solo e clima, é considerada a espécie que apresenta maior potencial para o combate a fome no mundo, desempenha papel estratégico tanto no aspecto econômico quanto social (ALONÇO et al., 2005).

A compatibilização da produção agrícola, conservação ambiental e a segurança alimentar tornaram-se o grande desafio deste século, o que tem como princípio a integração dos fatores biológicos nos sistemas de produção de arroz. A associação de bactérias promotoras de crescimento em gramíneas foi descrita com sucesso em cana-de açúcar, trigo, milho e arroz onde cultivares se desenvolveram sem suplementação elevada de adubo nitrogenado (RODRIGUEZ et al., 2003).

Esses bioestimulantes são também capazes de induzir resistência sistêmica através do aumento da atividade de enzimas (DALLAGNOL et al., 2005).

O objetivo do trabalho foi avaliar o efeito do silicato de cálcio e magnésio na produtividade do arroz em rotação com soja em sistema de plantio direto no estado do Maranhão.

\section{MATERIAL E MÉTODOS}

O experimento foi implantado em uma área cedida pelo Sindicato Rural de Imperatriz - Parque de Exposições.

Inicialmente realizou-se coleta e análise de solos para fins de fertilidade nos primeiros meses de execução do experimento.

Após os resultados da análise de solo, aplicou-se agrosilício nas dosagens de 0,1 , 2, 4, 8 t.ha $^{-1}$, e calcário para correção de acidez do solo na quantidade necessária de cálcio e magnésio para alcançar a maior dosagem do agrosilício.

A parcela foi dividida em duas subparcelas, as quais uma recebeu uma nova aplicação de agrosilício, e a outra permaneceu com a adubação residual da soja. Passados trinta dias, realizou-se a microbiolização das sementes com rizobactérias promotoras de crescimento 
(Pseudomonas sp. e Burlkoderia sp.) 24 horas antes do plantio.

O plantio foi conduzido em sistema de plantio direto, com parcelas de $3,50 \mathrm{~m} \mathrm{x}$ $12,00 \mathrm{~m}$.

A parcela foi subdividida em quatro sub-subparcelas, composta por dez linhas, em que cinco linhas eram compostas por sementes não microbiolizadas e cinco linhas com sementes microbiolizadas, uma subparcela com efeito residual do silicato de cálcio e magnésio, calcário aplicado no ano anterior e a outra com nova aplicação de silicato de cálcio e magnésio, calcário.

$\mathrm{O}$ esquema experimental foi de blocos ao acaso com parcelas sub-subdivididas com quatro repetições e cinco tratamentos, contendo diferentes dosagens de agrosilício $\left(0,1,2,4,8\right.$ t.ha $\left.{ }^{-1}\right)$ e semente microbiolizadas e não microbiolizadas com rizobactérias. A variedade de arroz utilizada foi a BRS Primavera.

$\mathrm{Na}$ área do plantio, foram analisados vários fatores: sementes microbiolizadas e não microbiolizadas; nova adubação com agrosilício e adubação residual do agrosilício.

Na avaliação considerou-se: Taxa de germinação (na área foram feitas contagens manuais com intervalos de 5 (cinco) dias para ter uma boa representatividade de germinação na área.
As análises de emergência foram realizadas de forma manual no campo, com contagens de intervalos de 5, 10 e 15 dias após o plantio.

As avaliações de alturas de plântulas foram feitas conforme a metodologia proposta por Marchezan (2001), onde estabelecia que a primeira avaliação fosse feita em paralelo com a primeira contagem de germinação.

Os dados de produtividade do arroz foram coletados em uma área útil de 1,5 $\mathrm{m}^{2}$ por sub-subparcelas eliminando-se as bordaduras. Esses dados foram coletados com cerca de 120 dias após o plantio.

Para avaliação da biomassa, foram coletadas plantas em $0,5 \mathrm{~m}^{2}$ das três linhas centrais/sub-subparcelas aos 60 dias após a semeadura e as plantas foram secas em estufa de circulação forçada a $70^{\circ} \mathrm{C}$ por três dias.

Os dados de massa de arroz (100 grãos cheios) foram coletados em uma área útil de $1,5 \mathrm{~m}^{2}$ por sub-subparcelas eliminandose as bordaduras.

Os dados foram submetidos a análises de variância (ANOVA) pelo programa ASSISTAT.

\section{RESULTADOS E DISCUSSÃO}

Os dados analisados mostram que não existem relações funcionais entre as variáveis adubação e microbiolização de sementes. De acordo com a tabela 1, as 
dosagens de agrosilício aplicadas na área e a adubação residual da soja em conjunto com a microbiolização de sementes com rizobactérias expressaram efeito negativo quanto ao crescimento de plântulas de arroz.

Tabela 1. Avaliação do crescimento de plântulas de arroz (Oryza sativa L.) submetidas a diferentes dosagens de silicato de cálcio e magnésio - agrosilício.

\begin{tabular}{|c|c|c|c|c|c|c|c|}
\hline Tratamentos & 0 t.ha ${ }^{-1}$ & 1 t.ha $^{-1}$ & 2 t.ha $^{-1}$ & 4 t.ha $^{-1}$ & 8 t.ha $^{-1}$ & Equação de regressão & $\mathbf{R}^{2}$ \\
\hline $\begin{array}{l}\text { Nova adubação } \\
\text { (S.M.) }\end{array}$ & $11,65 \mathrm{aA}$ & $11,75 \mathrm{aA}$ & $12,25 \mathrm{aA}$ & $11,65 \mathrm{aA}$ & $12,50 \mathrm{aA}$ & $y=11,6875+0,0875 x$ & 0,3010 \\
\hline $\begin{array}{l}\text { Nova adubação } \\
\text { (S.N.M.) }\end{array}$ & $12,25 \mathrm{aA}$ & $11,87 \mathrm{aA}$ & $12,25 \mathrm{aA}$ & $12,25 \mathrm{aA}$ & $12,00 \mathrm{aA}$ & $y=12,1625+(-0,0125) x$ & 0,2667 \\
\hline $\begin{array}{l}\text { Adubação } \\
\text { residual (S.M.) }\end{array}$ & $14,75 \mathrm{aA}$ & $14,50 \mathrm{aA}$ & $15,50 \mathrm{aA}$ & $14,75 \mathrm{aA}$ & $16,00 \mathrm{aA}$ & $y=14,6500+0,1500 x$ & 0,4286 \\
\hline $\begin{array}{l}\text { Adubação } \\
\text { residual } \\
\text { (S.N.M.) }\end{array}$ & $15,00 \mathrm{aA}$ & $15,25 \mathrm{aA}$ & $14,75 \mathrm{aA}$ & $15,75 \mathrm{aA}$ & $15,00 \mathrm{aA}$ & $y=15,1125+0,0125 x$ & $-0,3188$ \\
\hline $\mathrm{CV}(\%)$ & $45,87 \%$ & & & & & & \\
\hline
\end{tabular}

Médias seguidas de mesma letra, minúscula entre linhas e maiúsculas entre colunas, não diferem entre si pelo teste de Tukey a 5\%. S.M - Sementes microbiolizadas; S.N.M - Sementes não microbiolizadas.

Quando relacionado à biomassa da plântula de arroz (Tabela 2), as doses de agrosilício (adubação) e a microbiolização não promoveu aumento na biomassa da plântula. As variáveis analisadas (dosagens de agrosilício e microbiolização) não apresentaram efeito significativo para promoção de biomassa para a cultura do arroz em sistema de plantio direto. $\mathrm{O}$ alto valor do coeficiente de variância constatado para o fator analisado inviabiliza o experimento.

De acordo com o parâmetro analisado não houve efeito significativo com relação à massa de 100 grãos cheios, ou seja, na produção de grãos de arroz, não houve efeito da adubação e da microbiolização com rizobactérias (Tabela 3). De acordo com Barbosa-Filho e Prabhu (2002), em experimentos de avaliação de silicato de cálcio para a cultura do arroz, têm-se observado efeitos altamente positivos das doses desse componente na concentração de Si da palha e casca dos grãos de arroz, com aumentos de mais de $100 \%$ em relação ao tratamento sem esta adição. Efeitos estes não constados no experimento. Os dados também apontam um alto valor de coeficiente de variância, mostrando as diversas condições expressas ao experimento, como a declividade do terreno, incidência de insetos-praga, alta precipitação no local, dentre outros fatores. 
Tabela 2. Avaliação da biomassa $\left(\mathrm{g} . \mathrm{m}^{-2}\right)$ de plântulas de arroz (Oryza sativa L.) submetidas a diferentes dosagens de silicato de cálcio e magnésio - agrosilício.

\begin{tabular}{|c|c|c|c|c|c|c|c|}
\hline Tratamentos & 0 t.ha $^{-1}$ & 1 t.ha $^{-1}$ & 2 t.ha $^{-1}$ & 4 t.ha $^{-1}$ & 8 t.ha $^{-1}$ & Equação de regressão & $\mathbf{R}^{2}$ \\
\hline $\begin{array}{l}\text { Nova } \\
\text { adubação } \\
\text { (S.M.) }\end{array}$ & $0,10 \mathrm{aA}$ & $0,10 \mathrm{aA}$ & $0,55 \mathrm{aA}$ & $0,18 \mathrm{aA}$ & $0,16 \mathrm{aA}$ & $y=0,2233+(-0,0017) x$ & $-0,3322$ \\
\hline $\begin{array}{l}\text { Nova } \\
\text { adubação } \\
\text { (S.N.M.) }\end{array}$ & $0,26 \mathrm{aA}$ & $0,29 \mathrm{aA}$ & $0,58 \mathrm{aA}$ & $0,40 \mathrm{aA}$ & $0,15 \mathrm{aA}$ & $y=0,3953+(-0,0198) x$ & 0,1470 \\
\hline $\begin{array}{l}\text { Adubação } \\
\text { residual } \\
\text { (S.M.) }\end{array}$ & $0,16 \mathrm{aA}$ & $0,10 \mathrm{aA}$ & $0,47 \mathrm{aA}$ & $0,10 \mathrm{aA}$ & $0,10 \mathrm{aA}$ & $\mathrm{y}=0,2273+(-0,0138) \mathrm{x}$ & $-0,2359$ \\
\hline $\begin{array}{l}\text { Adubação } \\
\text { residual } \\
\text { (S.N.M.) }\end{array}$ & $0,35 \mathrm{aA}$ & $0,25 \mathrm{aA}$ & $0,10 \mathrm{aA}$ & $0,20 \mathrm{aA}$ & $0,26 \mathrm{aA}$ & $y=0,2433+(-0,0037) x$ & $-0,3109$ \\
\hline CV (\%) & $159,39 \%$ & & & & & & \\
\hline
\end{tabular}

Médias seguidas de mesma letra, minúscula entre linhas e maiúsculas entre colunas, não diferem entre si pelo teste de Tukey a 5\%. S.M - Sementes microbiolizadas; S.N.M - Sementes não microbiolizadas.

Tabela 3. Avaliação da massa de 100 grãos cheios $\left(\mathrm{g} \cdot \mathrm{m}^{-2}\right.$ ) de plântulas de arroz (Oryza sativa L.) submetidas a diferentes dosagens de silicato de cálcio e magnésio - agrosilício.

\begin{tabular}{|c|c|c|c|c|c|c|c|}
\hline Tratamentos & 0 t.ha' ${ }^{-1}$ & 1 t.ha $^{-1}$ & 2 t.ha $^{-1}$ & 4 t.ha $^{-1}$ & 8 t.ha $^{-1}$ & Equação de regressão & $\mathbf{R}^{2}$ \\
\hline $\begin{array}{l}\text { Nova } \\
\text { adubação } \\
\text { (S.M.) }\end{array}$ & $0,10 \mathrm{aA}$ & $0,10 \mathrm{aA}$ & $0,10 \mathrm{aA}$ & $0,10 \mathrm{aA}$ & $0,10 \mathrm{aA}$ & $y=x$ & - \\
\hline $\begin{array}{l}\text { Nova } \\
\text { adubação } \\
\text { (S.N.M.) }\end{array}$ & $1,05 \mathrm{aA}$ & $0,95 \mathrm{aA}$ & $1,93 \mathrm{aA}$ & $0,10 \mathrm{aA}$ & $0,97 \mathrm{aA}$ & $y=1,1395+(-0,0485) x$ & $-0,2586$ \\
\hline $\begin{array}{l}\text { Adubação } \\
\text { residual } \\
\text { (S.M.) }\end{array}$ & $0,10 \mathrm{aA}$ & $1,03 \mathrm{aA}$ & $0,10 \mathrm{aA}$ & $0,10 \mathrm{aA}$ & $0,10 \mathrm{aA}$ & $y=0,4255+(-0,0465) x$ & - 0,1667 \\
\hline $\begin{array}{l}\text { Adubação } \\
\text { residual } \\
\text { (S.N.M.) }\end{array}$ & $1,03 \mathrm{aA}$ & $0,10 \mathrm{aA}$ & $1,08 \mathrm{aA}$ & $0,10 \mathrm{aA}$ & $1,90 \mathrm{aA}$ & $y=0,4498+0,1308 x$ & $-0,0609$ \\
\hline $\mathrm{CV}(\%)$ & $292,61 \%$ & & & & & & \\
\hline
\end{tabular}

Médias seguidas de mesma letra, minúscula entre linhas e maiúsculas entre colunas, não diferem entre si pelo teste de Tukey a 5\%. S.M - Sementes microbiolizadas; S.N.M - Sementes não microbiolizadas.

A aplicação de agrosilício nas diferentes dosagens e a adubação residual da soja, bem como a microbiolização das sementes não refletiram em aumento de produtividade do arroz, nas condições em que foi realizado $\mathrm{o}$ experimento. $\mathrm{O}$ coeficiente de variância do experimento apresentou-se alto, indicando as condições adversas no experimento, o que levou a perdas de parcelas dificultando-se assim o desenvolvimento das plântulas na área, impossibilidade a execução do mesmo com êxito (Tabela 4). Possivelmente o efeito de Silício em características vegetativas da 
planta são fatores indiretos, uma vez que os principais aspectos da atuação desse elemento estão relacionados à sua deposição nas paredes celulares
(CAIXETA et al., 2009). Contudo recomenda-se a realização de novos estudos para melhor avaliação destes parâmetros.

Tabela 4. Avaliação da produtividade $\left(\mathrm{g} \cdot \mathrm{m}^{-2}\right)$ da cultura do arroz (Oryza sativa L.) submetidas a diferentes dosagens de silicato de cálcio e magnésio - agrosilício.

\begin{tabular}{|c|c|c|c|c|c|c|c|}
\hline Tratamentos & 0 t.ha' ${ }^{-1}$ & 1 t.ha ${ }^{-1}$ & 2 t.ha $^{-1}$ & 4 t.ha $^{-1}$ & 8 t.ha $^{-1}$ & Equação de regressão & $\mathbf{R}^{2}$ \\
\hline $\begin{array}{l}\text { Nova } \\
\text { adubação } \\
\text { (S.M.) }\end{array}$ & $0,10 \mathrm{aA}$ & $0,10 \mathrm{aA}$ & $0,10 \mathrm{aA}$ & $0,10 \mathrm{aA}$ & $0,10 \mathrm{aA}$ & $y=x$ & - \\
\hline $\begin{array}{l}\text { Nova } \\
\text { adubação } \\
\text { (S.N.M.) }\end{array}$ & $0,97 \mathrm{aA}$ & $0,38 \mathrm{aA}$ & $3,40 \mathrm{aA}$ & $0,10 \mathrm{aA}$ & $0,34 \mathrm{aA}$ & $y=1,4333+(-0,1318) x$ & 0,2080 \\
\hline $\begin{array}{l}\text { Adubação } \\
\text { residual } \\
\text { (S.M.) }\end{array}$ & $0,10 \mathrm{aA}$ & $0,10 \mathrm{aA}$ & $0,10 \mathrm{aA}$ & $0,10 \mathrm{aA}$ & $0,10 \mathrm{aA}$ & $y=x$ & - \\
\hline $\begin{array}{l}\text { Adubação } \\
\text { residual } \\
\text { (S.N.M.) }\end{array}$ & $0,77 \mathrm{aA}$ & $0,10 \mathrm{aA}$ & $2,33 \mathrm{aA}$ & $0,20 \mathrm{aA}$ & $1,10 \mathrm{aA}$ & $\mathrm{y}=0,8480+0,0240 \mathrm{x}$ & 0,3234 \\
\hline $\mathrm{CV}(\%)$ & $350,54 \%$ & & & & & & \\
\hline
\end{tabular}

\section{CONCLUSÃO}

Nas condições em que foi realizado o experimento as diferentes dosagens de agrosilício e a adubação residual da soja encontradas na área, bem como a microbiolização das sementes de arroz, não promoveram aumento do crescimento de plântulas, biomassa, produtividade e massa de 100 grãos cheios.

\section{REFERÊNCIAS}

ALONÇO, A. S.; SANTOS, A. B.; GOMES, A. S.; GRUTZMACHER, A. S et al. Cultivo do arroz irrigado no Brasil. EMBRAPA clima temperado. Nov 2005, ISSN 1808-9207 (Versão eletrônica).
Disponível

em: http://sistemasdeproducao.cnptia.embrapa. br/Fontes

HTML/Arroz/ArrozIrrigadoBrasil/autores. htm>. Acesso em: 11 abr. 2013.

BARBOSA-FILHO, M.P.; PRABHU, A.S. Aplicação de silicato de cálcio na cultura do arroz. Santo Antônio de Goiás: Embrapa Arroz e Feijão, 2002. 4p. (Embrapa Arroz e Feijão. Circular Técnica, $51)$.

CAIXETA, A.M.; GONÇALVES, L.D.; VALLONE, H.S.; FARIA, J.P.B.; MATOS, N.M.S.; PEIREIRA, L.C. Efeitos da adubação com silicato de cálcio e magnésio sobre o comprimento e número de folhas da cebola implantada em um solo de cerrado. II Semana de Ciência e Tecnologia do IFMG campus Bambuí. 2009. 
DALLAGNOL, L. J.; BALARDINI, R. S.; MADALOSSO, $M$. et al. Efeito do controle químico das doenças foliares sobre a produção e qualidade de arroz. In: CONGRESSO BRASILEIRO DE ARROZ IRRIGADO, 4, 2005, Santa Maria. Anais... Santa Maria: UFSM, 2005. p. 511-513.

MARCHEZAN, E. MENEZES, N.L., SIQUEIRA, C.A. Controle da qualidade das sementes de arroz irrigado utilizadas em Santa Maria/RS. Ciência Rural. Santa Maria, v.31, n.3, p.375-379, 2001.

RODRIGUES, F.A.; VALE, F.X.R.; KORNDÖRFER, G.H.; PRABHU, A.S.; DATNOFF, L.E.; OLIVEIRA, A.M.A.; ZAMBOLIM, L. Influence of silicon on sheath blight of rice in Brazil. Crop Protection, v. 22, p.23-29, 2003. 